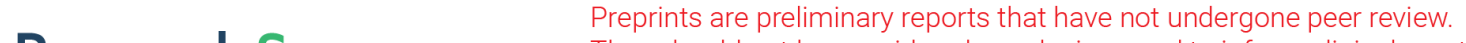 They should not be considered conclusive, used to inform clinical practice, or referenced by the media as validated information. \\ Exploration of Optimal Reconstruction Parameters for 18F-FDG Total-body PET/CT with Ultra-low Activity Injection
}

Xiuli Sui ( $\sim 19111210013 @ f u d a n . e d u . c n)$

Zhongshan Hospital Fudan University https://orcid.org/0000-0001-5065-4510

Hui Tan

Zhongshan Hospital Fudan University

Haojun Yu

Zhongshan Hospital Fudan University

Yiqiu Zhang

Zhongshan Hospital Fudan University

Pengcheng Hu

Zhongshan Hospital Fudan University

Jie Xiao

Zhongshan Hospital Fudan University

Chi Qi

Zhongshan Hospital Fudan University

Yuan Cheng

Zhongshan Hospital Fudan University

\section{Dai Shi}

Zhongshan Hospital Fudan University Hongcheng Shi

Zhongshan Hospital Fudan University

\section{Research Article}

Keywords: Reconstruction, Total-body PET/CT, Ultra-low Activity, Image quality, Quantitative analysis

Posted Date: April 27th, 2021

DOl: https://doi.org/10.21203/rs.3.rs-454119/v1

License: (1) (1) This work is licensed under a Creative Commons Attribution 4.0 International License.

Read Full License 


\section{Exploration of Optimal Reconstruction Parameters for ${ }^{18}$ F-FDG}

\section{Total-body PET/CT with Ultra-low Activity Injection}

Xiuli Sui, $\mathrm{MD}^{1,2,3^{*}}$, Hui Tan, MD, $\mathrm{PhD}^{1,2,3^{*}}$, Haojun Yu, BS ${ }^{1,2,3}$, Yiqiu Zhang, MD, $\mathrm{PhD}^{1,2,3}$, Pengcheng Hu, MD, $\mathrm{PhD}^{1,2,3}$, Jie Xiao, MD ${ }^{1,2,3}$, Chi Qi, MD ${ }^{1,2,3}$, Yuan Cheng, $\mathrm{MD}^{1,2,3}$, Dai Shi MD ${ }^{1,2,3}$, Hongcheng Shi, MD, $\mathrm{PhD}^{1,2,3 \#}$

1. Department of Nuclear Medicine, Zhongshan Hospital, Fudan University, Shanghai, 200032, China;

2. Nuclear Medicine Institute of Fudan University, Shanghai, 200032, China;

3. Shanghai Institute of Medical Imaging, Shanghai, 200032, China;

*Equally contributed to this work.

\#Corresponding author:

Hongcheng Shi, MD, PhD

Department of Nuclear Medicine

Zhongshan Hospital, Fudan University

180 Fenglin Road, Shanghai, China, 200032

Tel: 0086-21-64041990\#2064

Fax: 0086-21-64041990\#2064

Email: shi.hongcheng@zs-hospital.sh.cn 


\begin{abstract}
PURPOSE To explorer the optimal reconstruction parameters in oncologic ${ }^{\mathbf{1 8}} \mathbf{F}$-FDG total-body PET/CT studies with ultra-low activity injection.

METHODS A total of 204 reconstructed PET images of 34 patients with a total of 58 lesions were analyzed by two experienced nuclear medicine physicians. Images were reconstructed with ordered subset expectation maximization (OSEM) algorithm (2 and 3 iterations) including time-of-flight (TOF) and point spread function (PSF) corrections and regularization ordered subset expectation maximization (ROSEM) ( $\beta$-values of $0.3,0.4,0.5$, and 0.6 ). General image quality was assessed using the five-point method including overall image quality, image clarity, noise, and lesion conspicuity. Image noise, signal-to-noise ratio, lesion size, SUVmax, SUVpeak and $\mathrm{T} / \mathrm{N}$ were quantitatively analyzed by the third reader who did not participate in subjective image assessment.
\end{abstract}

\title{
RESULTS
}

In objective image quality indicators, noise decreased and a continuous increase of SNR with incremental $\beta$-values $(0.3,0.4,0.5$ and 0.6$)$ compared with OSEM3. In subjective image quality, OSEM2, ROSEM0.5 and ROSEM0.6 scored higher (all $\mathrm{P}<0.001)$ in overall image quality, image contrast and noise. The scores of ROSEM reconstructions were all higher in lesion conspicuity compared with OSEM3 (all $\mathrm{P}<0.001)$. In lesion detectability, SUVmax, SUVpeak and $\mathrm{T} / \mathrm{N}$ increase with $\beta$ value of ROSEM increase. Compared with OSEM3, there was a negative correlation between lesion size and the percentage increase of SUVpeak in OSEM2 and ROSEM 
reconstructions (all $\mathrm{P}<0.01$ ).

\section{CONCLUSION}

In clinical practice, we recommended OSEM reconstruction with 3 iterations with a relatively short reconstruction time and we recommend ROSEM algorithm with $\beta$ of 0.5 when reconstruction time is not considered.

\section{KEWORDS}

Reconstruction; Total-body PET/CT; Ultra-low Activity; Image quality, Quantitative analysis. 


\section{Introduction}

Current clinical PET/CT scanners cover an axial range of $15-30 \mathrm{~cm}$. The signal acquisition efficiency can be greatly improved by extending the axial range of the scanner. Subsequently, first total body PET scanner with a longitudinal field of view of $194 \mathrm{~cm}$ was brought up to provide up to 40 times the effective count rate than 22 cm AFOV scanners for total-body applications ${ }^{[1,2]}$. The imaging gain is about 4 times. And these gains have been proven to reduce injection dose or acquisition time or both and improve image quality ${ }^{[3,4,5]}$. It is significant for achieving low-dose scanning. We have previously demonstrated total-body dynamic PET imaging using $10 \times$ reduction of injected activity achieved comparable image contrast with full-activity imaging ${ }^{[6]}$. However, studies on the static image quality of activity reduction are not comprehensive. In clinical application, it is still a challenge whether the collected information can be transformed into visual images due to individual differences of patients and various factors. How to establish the optimal image reconstruction parameters is also a specific problem that needs to be solved urgently in clinical practice.

Ordered subset expectation maximization (OSEM) is the most commonly used image reconstruction method in PET clinical practice. The main disadvantage is that the noise increases with the number of iterations which image is unacceptable in clinical. If the iteration is too low, the contrast recovery is insufficient, and the lesions cannot be well displayed. Therefore, a trade-off needs to be made between image noise and quantitative accuracy which resulting insufficient image convergence ${ }^{[7,8]}$. In ultra-low 
activity injection, the count is smaller than that of full activity injection which resulting in higher noise. The regularized iterative algorithm developed by United Imaging compensates for this shortcoming to achieve full convergence. Noise control of the regularized iterative algorithm sinks into each iteration, and then finds the maximum likelihood solution through repeated iteration, so the image noise can be more significantly suppressed, but there is a risk of lesions being smoothed out. This complex algorithm includes a penalty function, managed by different parameters but only the $\beta$ factor is accessible which allows to control the noise which remains to be explored. In addition, the images information obtained by total-body PET/CT is extremely huge, reconstruction time is also a problem that needs to be considered. Therefore, the purpose of this study is to explore the influence of reconstruction algorithms on image quality by total body PET/CT with ultra-low activity injection and to find optimal reconstructions.

\section{Methods}

\section{Patients and image acquisition}

We retrospectively reviewed the patients who underwent total-body ${ }^{18} \mathrm{~F}-\mathrm{FDG} \mathrm{PET} / \mathrm{CT}$ with ultra-low activity injection $(0.37 \mathrm{MBq} / \mathrm{kg})$ in Zhongshan Hospital Fudan University from May 1st, 2020 to October 31, 2020. Patients with malignant tumor, providing histological confirmation, were eligible for analysis. Eventually, 34 patients who had a baseline pre-operative ${ }^{18}$ F-FDG PET/CT scan with sinogram data available for reconstruction were included in our study. 29 of them received surgical treatment, and 5 underwent aspiration biopsy. The characteristics of the patients were 
summarized in Table 1.

Patients were required to fast at least 6 hours and avoid strenuous exercise prior to ${ }^{18}$ F-FDG PET/CT imaging. The fasting blood glucose level was less than 7.1 mmol/L. Patients received an injection of ${ }^{18} \mathrm{~F}-\mathrm{FDG}$ according to their body weight (0.37 MBq/kg). ${ }^{18}$ F-FDG was provided by Shanghai Atom Kexing Pharmaceutical Co. Ltd and its radiochemical purity was more than $95 \%$. All patients rested quietly for approximately 60 minutes after the injection of ${ }^{18} \mathrm{~F}-\mathrm{FDG}$ and then underwent PET/CT. All patients were imaged using a PET/CT scanner with $194 \mathrm{~cm}$ view (uEXPLORER, United Imaging Medical Group, Shanghai, China). The PET data was obtained through 3D list mode, in which continuously collected for 15 minutes.

\section{Image processing}

A total of 5 patients were evaluated in the pre-analysis, and raw data sets were reconstructed with following 8 different reconstruction protocols. OSEM reconstruction with 2, 3 and 4 iterations, regularized ordered subset expectation maximization ROSEM (HYPER Iterative, United Imaging Healthcare, Shanghai, China) with incremental $\beta$-values of $0.1,0.3,0.5,0.7,1$ respectively. The results show that the reconstructions are better when OSEM reconstruction with 2 and 3 iterations, and ROSEM reconstruction with $\beta$ values of 0.3 and 0.5 (the data is not shown). Thus, for further detailed analysis, two sets using OSEM reconstruction protocol with time-of-flight (TOF) and point spread function (PSF) were reconstructed respectively ( 2 and 3 iterations, 20 subsets, matrix 192*192, slice thickness $1.443 \mathrm{~mm}$, and the full width at half maximum of the Gaussian filter function $3 \mathrm{~mm}$ ) which substitute as 
OSEM2 and OSEM3 later. For comparison, ROSEM reconstruction protocal with $\beta$ of $0.3,0.4,0.5$, and 0.6 were reconstructed (represented as ROSEM0.3, ROSEM0.4, ROSEM0.5, ROSEM0.6 next). The CT scan parameters were as follows: tube voltage $120 \mathrm{kV}$, tube current $140 \mathrm{mAs}$, pitch 1.0 , collimation $0.5 \mathrm{~mm}$, and reconstructed slice thickness $0.5 \mathrm{~mm}$.

In ROSEM, the penalized likelihood function is written as follows:

$$
\mathrm{D}=\operatorname{argmax}_{f \geq 0} \sum_{i j}-a_{i j} f_{j}+c_{i j} \log \left(a_{i j} f_{j}\right)-\gamma \cdot U(f)
$$

c represents the PET coincidence data obtained by detection, $\mathrm{f}$ represents the estimated image, a represents the system matrix, i represents the projection index, and $\mathrm{j}$ represents the image pixel index. Among them, $U(f)$ is the penalty function used to control the noise, and $\gamma$ is the parameter used to adjust the noise level.

$$
\gamma \cdot U(f)=\beta \cdot g\left(N E C, s n s_{j}\right) \cdot U(f)
$$

The parameter modeling uses global factor, local factor and penalty intensity $\beta$. Global factor characterizes noise equivalent counts (NEC) influence items, which can compensate for low count or low-quality data. The local factor compensates for changes in spatial sensitivity caused by system geometry and changes in noise level caused by attenuation. The penalty intensity $\beta$ provides the user with an open and adjustable parameter. This parameter is the only parameter that the user needs to adjust. It can be adjusted in the interval of $(0,1]$. It has embedded TOF and PSF technologies, which can adaptively adjust the number of iterations and the number of subsets to find the optimal solution.

\section{Image evaluation}


A total of 204 reconstructed PET data sets (34 patient studies 58 lesions with 6 different reconstructions) were evaluated in random order by two experienced nuclear radiologists (with 6 and 7 years of experience interpreting PET/CT respectively) blinded to the reconstruction method used. The two readers subjectively evaluated image quality of each PET data using a five-point scale by overall image quality, image clarity, noise, and lesion conspicuity. The criteria used for these grades are summarized in Table 2 and are based on previously published studies assessing image quality ${ }^{[9,10]}$. All positive lesions of PET with pathologically confirmed were analyzed, with a maximum of 6 lesions selected per patient. If there are more than 6 lesions in one site, 6 target lesions (the largest and smallest three lesions based on size) are defined for further analysis in order to explore the detection capabilities of small lesions.

Lesion size, SUVmax and SUVpeak were measured by a third reader who did not participate in subjective image assessment. The longest diametral line of the lesion indicates the lesion size in our study. A round-shaped $(1 \mathrm{~cm}$ diameter $)$ region of interest (ROI) was placed in four homogeneous area of the liver (avoiding intrahepatic lesions and larger blood vessels), the spleen (hilar level), the second lumbar spine, and the bilateral gluteus maximus. The maximum standard uptake value (SUVmax), average standard uptake value (SUVmean) and standard deviation (SD) were recorded for each ROI. SD is defined as the noise and the signal-to-noise ratio (SNR) is calculated by dividing SUVmean in ROI by its SD. SUVmax and SUVmean of ascending aorta (bronchial bifurcation) were recorded. The ROI of all reconstructed 
images is drawn synchronously in 2D mode to ensure that each ROI is the same location and size. The VOI of each lesion was placed to measure SUVmax, SUVpeak. The tumor background ratio $(\mathrm{T} / \mathrm{N})$ was calculated by dividing the lesion SUVmax by the SUVmean of the blood pool.

\section{Statistical analysis}

Microsoft Excel and SPSS 22.0 Windows software (IBM SPSS Inc., Armonk, New York, USA) were used for statistical analysis. Continuous variables are expressed as mean \pm standard deviation (SD). Since objective image quality of each reconstruction were distributed normally, statistical differences were assessed using repeated measures analysis of variances (ANOVA) with post hoc Bonferroni corrections to adjust for multiple comparisons. Qualitative image ratings and quantitative SUVmax, SUVpeak and T/N values were analyzed with the Friedman test separately. Weighted Kappa was used to test the consistency among the subjective raters. The differences in SUVmax, SUVpeak, and T/N between reconstructions of each patient were calculated, and the mean value and SD of these differences were then calculated for all patients. A P value $\leq 0.05$ is considered significant.

\section{Results}

\section{Patient characteristics}

We enrolled 34 patients (16 females, 18 males) with an average age of 59.0 \pm 12.2 years and a body mass index (BMI) of $22.1 \pm 1.6 \mathrm{~kg} / \mathrm{m}^{2}$ (range $\left.19.04-24.74 \mathrm{~kg} / \mathrm{m}^{2}\right)$. The dose of FDG injected was $23.73 \pm 2.83 \mathrm{MBq}$ (range $18.46-30.34 \mathrm{MBq}$ ). Details are listed in Table 1. 


\section{Objective image quality}

The results of the quantitative analysis including SNR and noise in liver, spleen, bone and muscle of different reconstructions are given in Table 2. Of all the observations, noise decreased and a continuous increase of SNR with incremental $\beta$-values $(0.3,0.4$, 0.5 and 0.6) compared with OSEM3. There are significantly differences among OSEM3 and OSEM2, ROSEM0.5, ROSEM0.6 (all p<0.001), no significant difference with ROSEM0.3.

\section{Subjective image quality}

OSEM2, ROSEM0.5 and ROSEM0.6 scored higher comparing with OSEM3 in overall image quality. Similar differences were observed for image contrast and noise (all $\mathrm{p}<0.001$ ). In lesion conspicuity, ROSEM reconstructions scored higher comparing with OSEM reconstructions $(\mathrm{P}<0.001)$ (Table 4).

\section{Lesion detectability}

Table 3 shows the SUVmax, SUVpeak, T/N mean and SD of 58 tumor lesions by 6 reconstructions. The comparison results are shown in Figure2. As $\beta$ value of ROSEM increase, all observations increase. OSEM2 was lower and ROSEM0.3, ROSEM0.4, ROSEM0.5 were higher than OSEM3 among all the observed values (all $\mathrm{P}<0.05$ ). The relative SUVmax, SUVpeak and $\mathrm{T} / \mathrm{N}$ of $\triangle$ ROSEM0.3, $\Delta$ ROSEM0.4, $\triangle$ ROSEM0.5 and $\triangle$ ROSEM0.6 were significantly increased after standardization according to OSEM3. The median lesion size was $1.55 \mathrm{~cm}$ (range, 0.7-11). There was a negative correlation between lesion size and the percentage increase of lesion SUVpeak in the reconstructions standardized by OSEM3 (all $\mathrm{P}<0.01$ ), but no 
significance in SUVmax and T/N (Figure3).

Figure 4 shows typical images of the 6 reconstructions. ROSEM0.6 exhibited decreased percentage of liver lesions missed diagnosis when compared to that of OSEM2 $(1.7 \%, 1 / 58$ VS. 5.2\%, 3/58) in Figure 5.

\section{Discussion}

Our previous results have showed high quality images were achieved after $25 \mathrm{MBq}$ activity injection scanned for 10 minutes (20 subsets and 2 iterations reconstructed using OSEM-PSF-TOF) in one subject $(43.5 \mathrm{~kg}, 152 \mathrm{~cm})^{[3]}$. So, it is important to establish the optimal image reconstruction parameters in clinical application. In this study, we selected various reconstructions parameters to find influence on image quality of total-body ${ }^{18}$ F-FDG PET/CT with ultra-low activity injection and carried out quantitative analysis.

As shown in Table 2, the SNR of OSEM2, ROSEM0.5 and ROSEM0.6 in our study was higher and the noise was less comparing with OSEM3. This result is predictable, because the noise increase and the signal-to-noise ratio is worse with more iterations in OSEM reconstructions and noise was controlled with filtering after iterations ${ }^{[11]}$. While ROSEM adds the noise control process to each iteration. There is global factor which compensate for low counts or poor-quality data and local factor which compensates the variation of spatial sensitivity caused by the geometry of the system and the variation of noise level caused by attenuation in each iteration. Therefore, we believe that in terms of objective image quality, it is better to apply OSEM reconstruction with 2 iterations and ROSEM reconstruction with $\beta$ values of 0.5 and 
0.6 in total-body PET imaging with ultra-low activity injection.

As semi-quantitative PET parameters such as SUVmax have been reported as potential prognostic imaging markers for cancer patients ${ }^{[12]}$. And some treatment response assessment protocols recommend using the SUVpeak of the tumor, tumor-to-liver ratio and $\mathrm{T} / \mathrm{N}$ for treatment response assessment ${ }^{[13]}$. Accurate quantitative analysis is vital important in malignant tumor management. In our study, quantitative parameters such as SUVmax, SUVpeak and T/N evaluation of ROSEM reconstructions are also increase obviously compared with OSEM and the percentage increases to be higher for SUVmax and $\mathrm{T} / \mathrm{N}$ than for SUVpeak. The ROSEM reconstructions can demonstrate this parameter more accurately through effective convergence, which may increase their utility as biomarkers. In table3, lesions size was negatively correlated with the degree of SUVpeak evaluation which means small lesions were improved more significantly. SUVmax and T/N were also negatively correlated with lesions size but no statistical significance was detected. This was not consistent with our expectations. We speculated to be related to the heterogeneity of the lesions. ROSEM yields larger SUVmax increments in lesions with high tracer uptake and smaller increments in lesions with low tracer uptake (slight above background). Another reason may be the small number of lesions, larger patient trials are certainly needed to validate these initial results.

The grade results showed that manufacturer-recommended reconstruction OSEM3 have achieved clinical diagnosis. OSEM2, ROSEM0.5 and ROSEM0.6 scores were significantly improved compared with OSEM3. However, there were missed 
diagnosis in OSEM2 and ROSEM0.6 according to visual analysis and the minimal level of tumor uptake proposed in PERCIST $1.0^{[14]}$. It is reasonable detected in these liver lesions with the sensitivity of ${ }^{18} \mathrm{~F}-\mathrm{FDG}$ PET/CT is limited by liver background. To a certain extent, ROSEM reconstructions improve $\mathrm{T} / \mathrm{N}$ of liver lesions with reducing background noise and increasing SUVmax through multiple iterations. But as the beta value increases, the likelihood of lesions with low tracer uptake being smoothed increases, which may decrease the sensitivity of PET detection of liver lesions. Even though the image quality of OSEM2 and ROSEM0.6 are relatively good, the rate of missed diagnoses was unacceptable which are not recommended for clinical practice.

\section{Conclusion}

In ${ }^{18}$ F-FDG total-body PET/CT with ultra-low activity injection (acquisition time 15min), OSEM2 and ROSEM0.6 have good image quality but missed diagnoses of lesions which is unacceptable in clinical. OSEM3 has good diagnosis ability of lesions and the image quality have meets the clinical requirements. ROSEM0.5 reconstruction showed a relevant improvement in lesion conspicuity and image quality compared with OSEM reconstructions, but the reconstruction time is about 1.5 times that of OSEM3. Above all, we recommended OSEM algorithm to reconstruct 3 iterations with a relatively short reconstruction time. When reconstruction time is not considered, we recommend ROSEM algorithm with $\beta$ of 0.5 in clinical practice.

\section{Funding}

This study is supported by Science and Technology Committee of Shanghai 
Municipality (Grant No. 20DZ2201800 to Yiqiu Zhang), Special Fund for Clinical Research of Zhongshan Hospital, Fudan University (No.2020ZSLC63 to Yiqiu Zhang), and Shanghai Municipal Key Clinical Specialty (No. shslczdzk03401).

\section{Conflict of interest}

The authors declare that they have no conflict of interest.

\section{Availability of data and material}

The data that support the findings of this study are available from the corresponding author upon reasonable request.

\section{Authors' contributions}

Xiuli Sui and Hui Tan were involved in the study design, data analysis and manuscript preparation. Jie Xiao, Yuan Cheng and Dai Shi helped with data processing. Haojun Yu and Chi Qi helped with image acquisition and processing. Yiqiu Zhang and Pengcheng $\mathrm{Hu}$ helped with the revision of the manuscript and image quality evaluation. Hongcheng Shi designed the study and contributed to the data analsis and writing of the manuscript. This retrospective study of existing patient data and images was approved by the institutional review board of Zhongshan Hospital, Fudan University. The requirement for informed consent was waived.

\section{Ethical approval}

All procedures performed in studies involving human participants were in accordance with the ethical standards of the institutional and/or national research committee and with the 1964 Helsinki declaration and its later amendments or comparable ethical standards. 


\section{References}

1. Poon JK, Dahlbom ML, Moses WW, et al. Optimal whole-body PET scanner configurations for different volumes of LSO scintillator: a simulation study. Phys Med Biol. 2012;57:4077-4094.

2. Badawi RD, Poon JK, Surti S, et al. EXPLORER, an ultrasensitive total-body PET scanner: application feasibility simulations. Paper presented at: the World Molecular Imaging Congress, Savannah, Georgia, September 2013.

3. Badawi RD, Shi H, Hu P, et al. First Human Imaging Studies with the EXPLORER Total-Body PET Scanner. J Nucl Med. 2019 Mar;60(3):299-303. doi: 10.2967/jnumed.119.226498. Epub 2019 Feb 7. PMID: 30733314; PMCID: PMC6424228.

4. Zhang YQ, Hu PC, Wu RZ, et al. The image quality, lesion detectability, and acquisition time of 18F-FDG total-body PET/CT in oncological patients. Eur J Nucl Med Mol Imaging. 2020 Oct;47(11):2507-2515. doi: 10.1007/s00259-020-04823-w. Epub 2020 May 18. PMID: 32424483.

5. Tan H, Sui X, Yin H, et al. Total-body PET/CT using half-dose FDG and compared with conventional PET/CT using full-dose FDG in lung cancer. Eur J Nucl Med Mol Imaging. 2020 Nov 27. doi: 10.1007/s00259-020-05091-4. Epub ahead of print. PMID: 33244618.

6. Liu G, Hu P, Yu H, Tan H, Zhang Y, Yin H, Hu Y, Gu J, Shi H. Ultra-low-activity total-body dynamic PET imaging allows equal performance to full-activity PET imaging for investigating kinetic metrics of $18 \mathrm{~F}-\mathrm{FDG}$ in healthy volunteers. Eur $\mathrm{J}$ Nucl Med Mol Imaging. 2021 Jan 22.

7. Adams MC, Turkington TG, Wilson, JM, Wong TZ. A systematic review of the factors affecting accuracy of SUV measurements. Am J Roentgenol 2010;195:310-320.

8. Jaskowiak CJ, Bianco JA, Perlman SB, Fine JP. Influence of reconstruction iterations on 18F-FDG PET/CT standardized uptake values. J Nucl Med 2005; 46:424-428.

9. Messerli M, Stolzmann P, Egger-Sigg M, Trinckauf J, D'Aguanno S, Burger IA, von Schulthess GK, Kaufmann PA, Huellner MW. Impact of a Bayesian penalized likelihood reconstruction algorithm on image quality in novel digital PET/CT: clinical implications for the assessment of lung tumors. EJNMMI Phys. 2018 Sep 26;5(1):27. 10.van Sluis J, Boellaard R, Somasundaram A, et al. Image quality and semiquantitative measurements on the biograph vision PET/CT system: initial experiences and comparison with the biograph mCT. J Nucl Med. 2020;61(1):129-135.

11. Tong S, Alessio AM, Kinahan PE. Image reconstruction for PET/CT scanners: past achievements and future challenges. Imaging Med. 2010;2:529-545. doi: 10.2217/iim.10.49.

12. Yoh T, Seo S, Morino K, et al. Reappraisal of Prognostic Impact of Tumor SUVmax by $18 \mathrm{~F}-F D G-P E T / C T$ in Intrahepatic Cholangiocarcinoma. World J Surg. 2019 May;43(5):1323-1331. doi: 10.1007/s00268-019-04917-z. PMID: 30680501. 
13. Sarikaya I, Sarikaya A. Assessing PET Parameters in Oncologic 18F-FDG Studies. J Nucl Med Technol. 2020 Sep;48(3):278-282. doi: 10.2967/jnmt.119.236109. Epub 2019 Dec 6. PMID: 31811061.

14. Wahl RL, Jacene H, Kasamon Y, et al. From RECIST to PERCIST: Evolving Considerations for PET Response Criteria in Solid Tumors[J]. J Nucl Med, 2009,50(Suppl_1):122S-150S. DOI:10.2967/jnumed.108.057307.

\section{Tables}

Table 1. Clinical Data and Demographic of Patients $(n=34)$ Who Underwent ${ }^{18}$ F-FDG Total-body PET/CT with Ultra-low Activity Injection

Table 2. Subjective Image Quality-background

Table 3. Median Lesion SUVmax, SUVpeak and T/N Scores Derived from Images Obtained Using Different Reconstruction Settings

Table 4. Objective Image Quality-score 
Figures
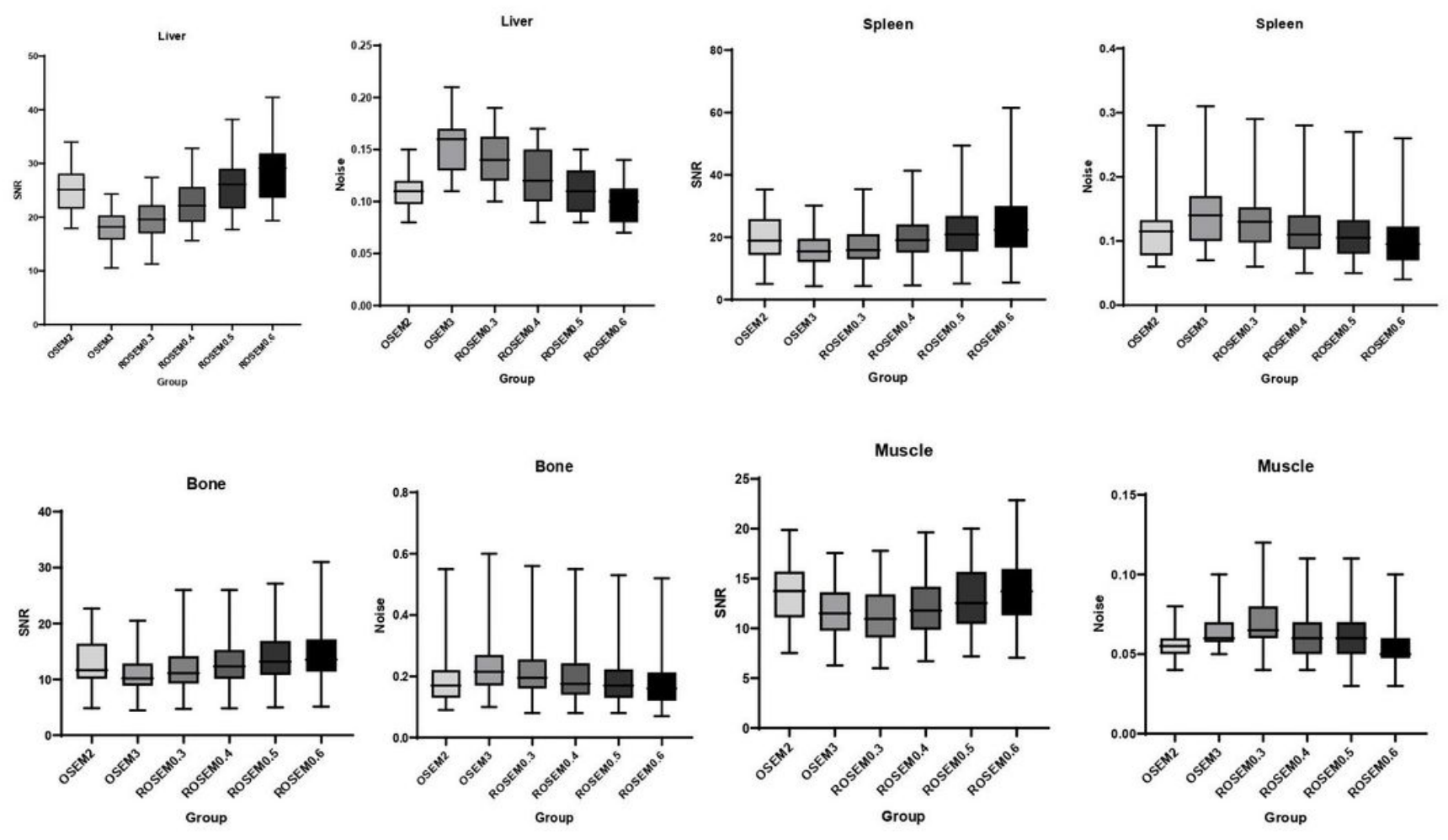

F1

Figure 1

Please see the Manuscript PDF file for the complete figure caption 

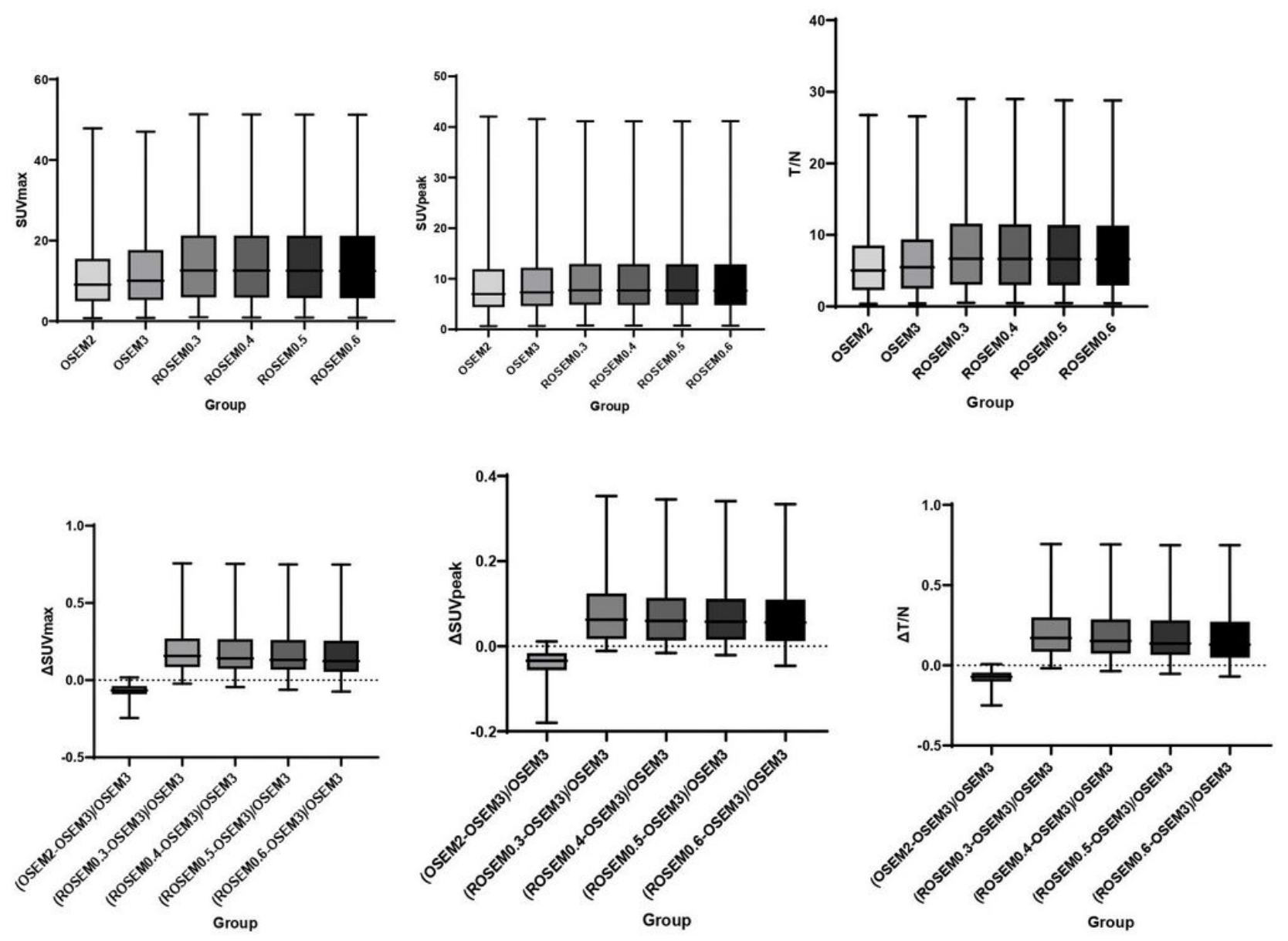

F2

Figure 2

Table 3 shows the SUVmax, SUVpeak, T/N mean and SD of 58 tumor lesions by 6 reconstructions. The comparison results are shown in Figure2.

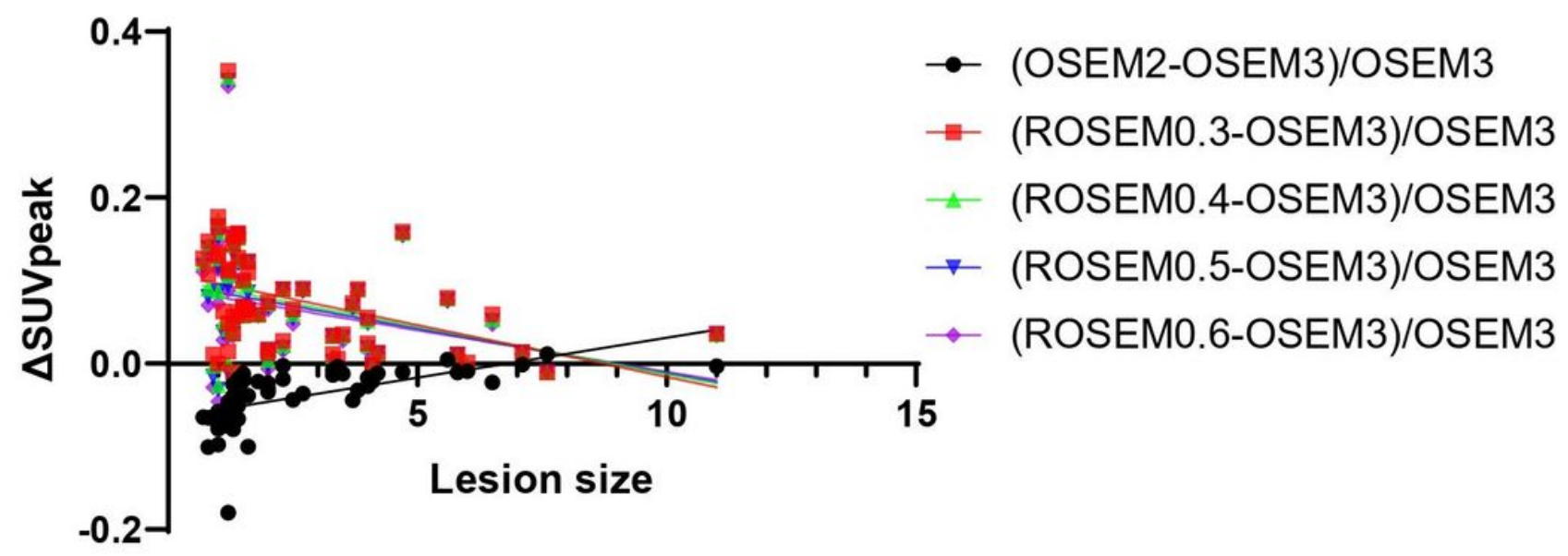


Figure 3

There was a negative correlation between lesion size and the percentage increase of lesion SUVpeak in the reconstructions standardized by OSEM3 (all $\mathrm{P}<0.01$ ), but no significance in SUVmax and $T / \mathrm{N}$ (Figure3).
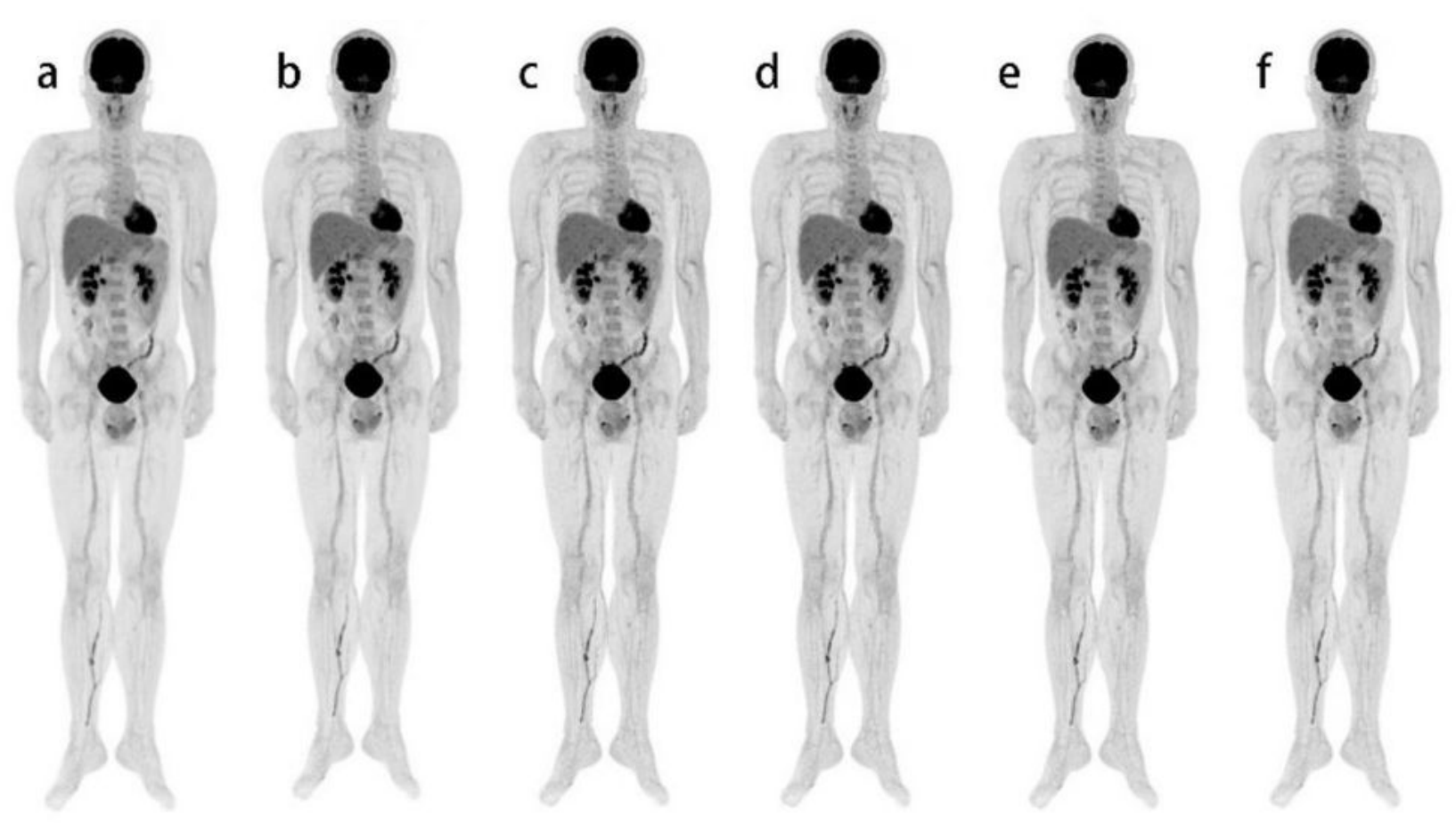

\section{g}

$\mathrm{h}$

i
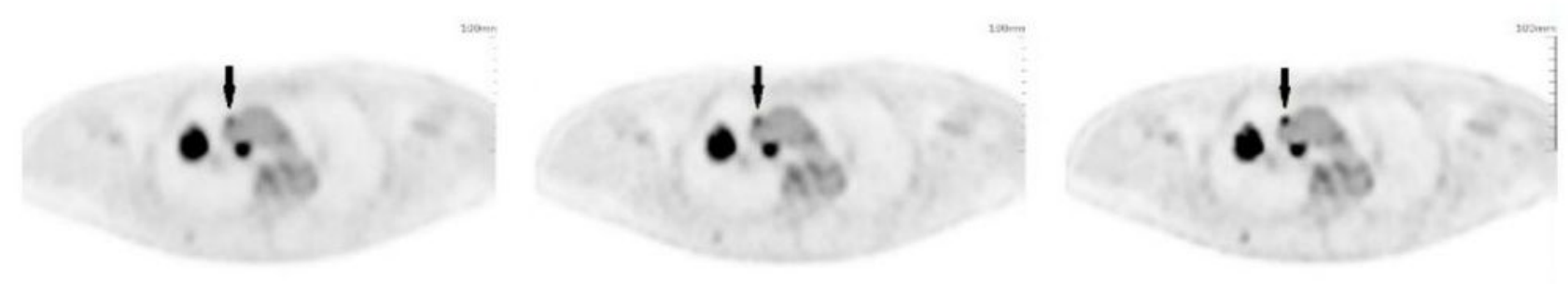

j

k

I
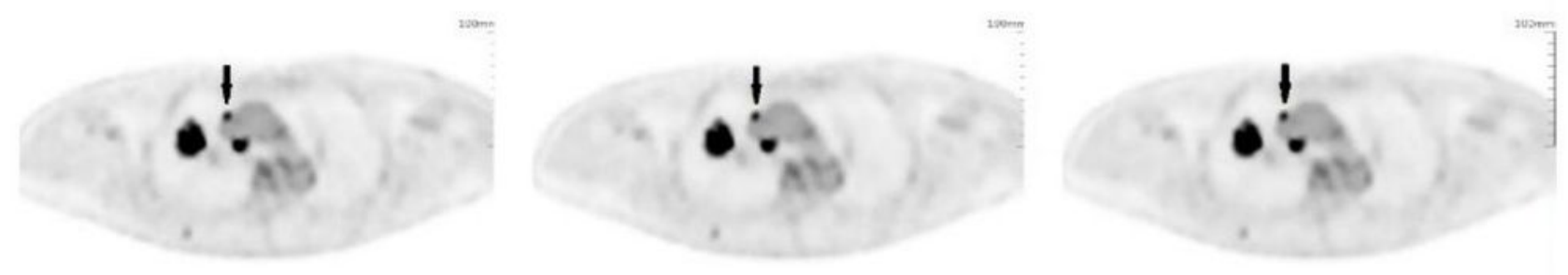

Figure 4 
(Top) Selections of MIPs from reconstructions of a 45-year-old man with pancreatic cancer confirmed by surgery(a-f) for OSEM2 to ROSEM0.6. The apparent noise decreases as $\beta$ value increased; all the images appear to be of diagnostic quality. (Bottom) Axial images of a 40-year-old woman with invasive lung adenocarcinoma confirmed by surgery(g-l) for OSEM2 to ROSEM0.6. PET showed avid FDG in right upper lobe and mediastinum lymph nodes. The arrow shows the significance of the lesion reconstructed by ROSEM.

m

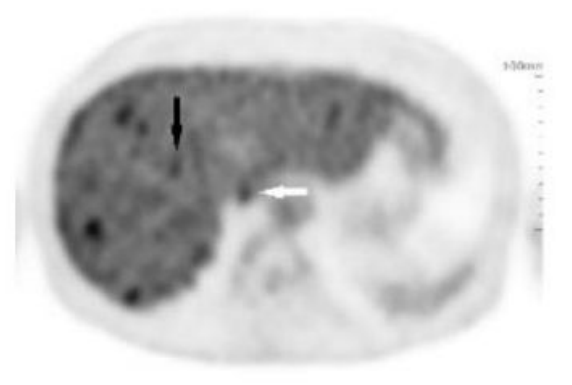

p

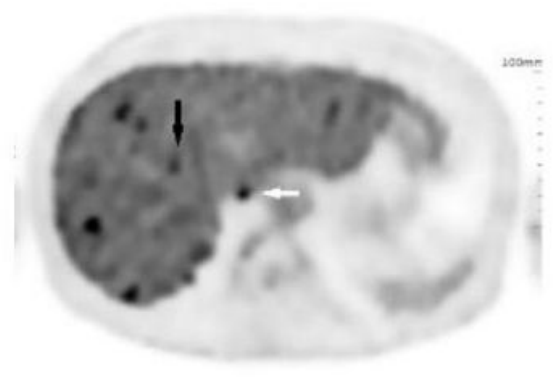

$\mathrm{n}$

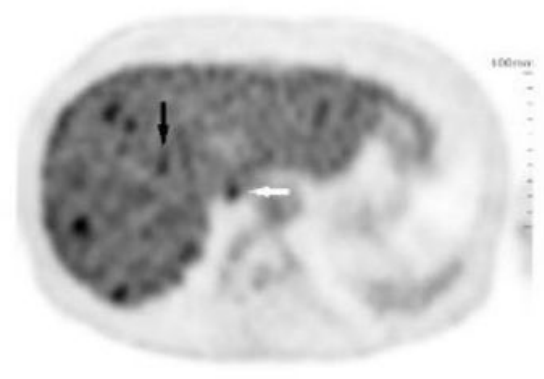

q

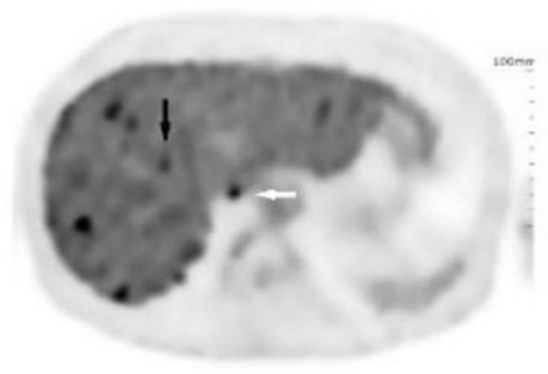

0

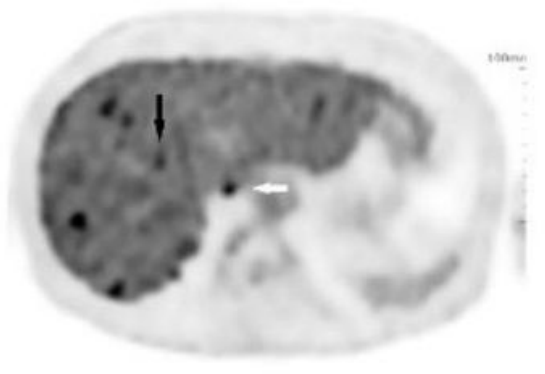

$r$

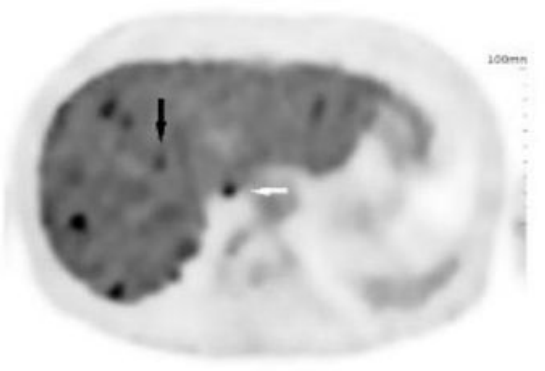

\section{Figure 5}

Axial images of a 71-year-old man with Hepatic metastasis of pancreatic carcinoma (m-r) for OSEM2 to ROSEM0.6. The lesion indicated by the white arrow was not obvious in $m(T / N=1.81)$. The lesions indicated by the black arrow were not obvious in $\mathrm{m}$ and $\mathrm{r}$, with $\mathrm{T} / \mathrm{N}$ of 1.91 and 1.95 , respectively. Moreover, they could not meet the minimal level of tumor uptake proposed in PERCIST 1.0

\section{Supplementary Files}

This is a list of supplementary files associated with this preprint. Click to download.

- renamed7c997.xIsx 